\title{
Human Vital Signs Monitor
}

\section{Tatiana Kelemenová ${ }^{1}$, Michal Kelemen ${ }^{1,}{ }^{*}$, Ondrej Benedik ${ }^{2}$, Ivana Koláriková ${ }^{1}$, Matúš Svrčina ${ }^{1}$, Filip Filakovský ${ }^{1}$, Lukáš Leštach ${ }^{1}$}

Technical University of Košice, Faculty of Mechanical Engineering, Letná 9, 04200 Košice, Slovak Republic

Kybernetes, s.r.0., Omska 14, Kosice, Slovak Republic

Abstract: The article deals with the analysis of human vital signs and the design of a low-cost device for monitoring these quantities for domestic use. The aim is to make such devices available for home use and preliminary diagnostics. The article presents the results of experiments with commercially available sensors designed to measure these quantities.

Keywords: vital sign; measurement; monitor; Arduino.

\section{Introduction}

In today's hectic world, a person is exposed to enormous stress and psychologically demanding situations that cause a rapid deterioration in a person's health. It is therefore important to have facilities for monitoring human health. The main idea of this work was to create a low-cost device that would perform the function of monitoring at least some vital functions such as body temperature, heart rate pulse, respiratory rate, blood pressure and blood oxygen saturation, etc. By monitoring these vital signs, it is possible to prevent many diseases and sudden death. Extensive monitoring is used mainly in hospital wards when hospitalizing a patient using specialized devices. However, the technologies used in these devices also penetrate the domestic sphere intended for the general population. The best examples are smartwatches and smartphones that can record the pulse and oxygenation of the blood with oxygen at the same time and measure other parameters. Our goal was to create a low-cost alternative to commercially available devices that would measure at least some of the basic vital signs of a human being.

Wearable vital signs monitoring system that joins textiles with microelectronics has been developed. It was designed and developed to be a usable practical approach for different clinical scenarios, in hospitals, home or on the move, that need continuous or frequent high quality vital signs monitoring from its wearer [1].

This paper [2] presents a wearable vital signs monitor at the ear. The monitor measures the electrocardiogram (ECG), ballistocardiogram (BCG), and photoplethysmogram (PPG) to obtain pre-ejection period (PEP), stroke volume (SV), cardiac output (CO), and pulse transit time (PTT). The ear is demonstrated as a natural anchoring point for the integrated sensing of physiological signals [2].

A patient surveillance system employing a wireless body-worn vital signs monitor with automated nursing notification of alarms via smartphones was used to gather data. Data collected included: alarm rates, rapid response team (RRT) calls, intensive care unit (ICU) transfers, and unplanned deaths before and during the pilot study [3].

A continuous, wearable and wireless vital signs monitor at the ear is demonstrated. The device has the form factor of a hearing aid and is wirelessly connected to a PC for data recording and analysis. The device monitors the electrocardiogram (ECG) in a single lead configuration, the ballistocardiogram (BCG) with a MEMS triaxial acceler-

*Corresponding author: Michal Kelemen, $\boldsymbol{E}$-mail address: michal.kelemen@tuke.sk 
ometer, and the photoplethysmograms (PPG) with $660 \mathrm{~nm}$ and $940 \mathrm{~nm}$ LED sources and a static photocurrent subtraction analogue front end [4].

In the paper [5], the system uses the vital signs monitoring devices to detect the body's vital signs for a long time, which mainly includes the heart rate, the breathing rate and the state of the bed. It can transfer to the cloud platform server through the WIFI, and the cloud platform server can screen, analyse and calculate vital signs data and the dynamic change of vital signs state. The person under monitoring can see the data of daily vital signs and the sleep state through the mobile terminal. Managers can see the changes of user's data and state to detect the change of vital signs momently [5].

\section{Quantities of human vital signs and tested modules for measuring human vital signs}

Vital signs such as body temperature, blood pressure, pulse (heart rate), and breathing rate (respiratory rate) are used to monitor human vital signs, and pulse oximetry is often added to determine blood oxygen saturation.

Pulse sensor - is designed to measure heart rate. It consists of an LED as a light transmitter and a photodiode as a light receiver. The LED emits green light with a wavelength of $565 \mathrm{~nm}$. The manufacturer states that it is possible to record the heart rhythm at the end of the finger or on the earlobe. The output from the sensor is an analogue signal (fig. 1) [6, 7].
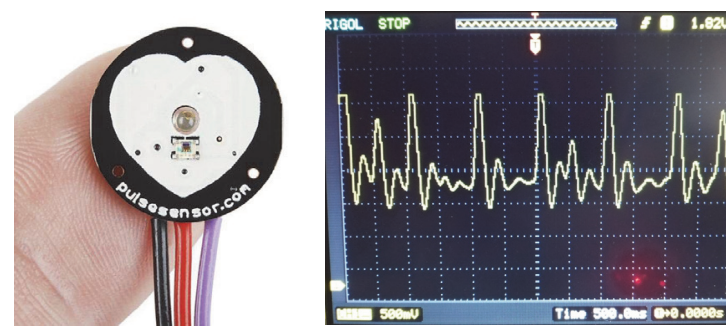

Figure 1: Pulse Sensor and time course of the output signal [6].

Pulse oximeter sensor - The sensor module contains the MAX30102 sensor (fig. 2) and other accessories. The MAX30102 module is an integrated oximeter and pulse sensor $[6,7]$.

There are two diodes and optimized optics on the chip. You can find similar sensors in, for example, SMART watches, fitness bracelets or sports trackers. The module supports software power-down and can therefore be used in applications requiring low consumption. The working supply voltage is in the range $1.8 \mathrm{~V}-3.3 \mathrm{~V}$. The output signal is solved using the I2C bus (fig. 2). The manufacturer does not provide a module diagram [7].
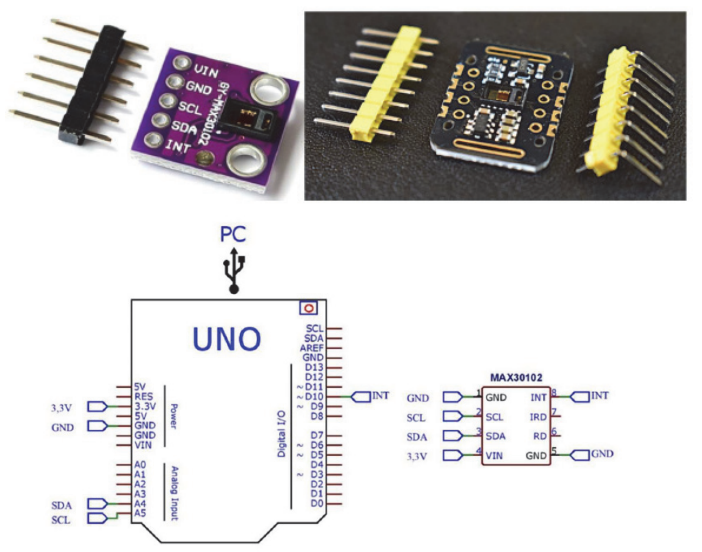

Figure 2: Tested pulse oximeter sensor modules and electrical connection of the module with MAX3102 sensor with Arduino UNO microcontroller [7].

Electrocardiography sensor - sensor (fig. 3) with integrated circuit AD8232 (Analog Devices) is an integrated circuit designed for measuring biosignals, especially for registration of single-lead electrocardiogram $[8,9]$. The chip is designed to extract, amplify and filter weak biosignals in the presence of interfering elements (human movement, imperfect electrode placement). Its primary applications include use in portable monitoring devices such as a heart rate fitness monitor and a portable electrocardiogram. For this reason, the chip size is also minimal $(4 \times 4 \mathrm{~mm})$. The power supply is solved by a direct voltage in the range of $2.0-3.5 \mathrm{~V}$. The current consumption is typically $170 \mu \mathrm{A}$, while it is also possible to switch to the shutdown mode, when the current consumption is only $100 \mathrm{nA}$ [9].

Other features of the chip are overvoltage protection at the input, integrated operational amplifiers, which can be used for active signal filtering. The chip can also be connected to a configuration for two-electrode sensing, where data is obtained only to measure the heart rate, not to record the electrocardiographic curve. It is also possible to detect the loss (disconnection) of the electrode by means of the true / false message on the output pins 11, 12 (fig. 3) [9].

Our module with the AD8232 chip is built according to the datasheet as a heart rate monitor 
to observe the shape of the electrocardiographic curve (fig. 3).
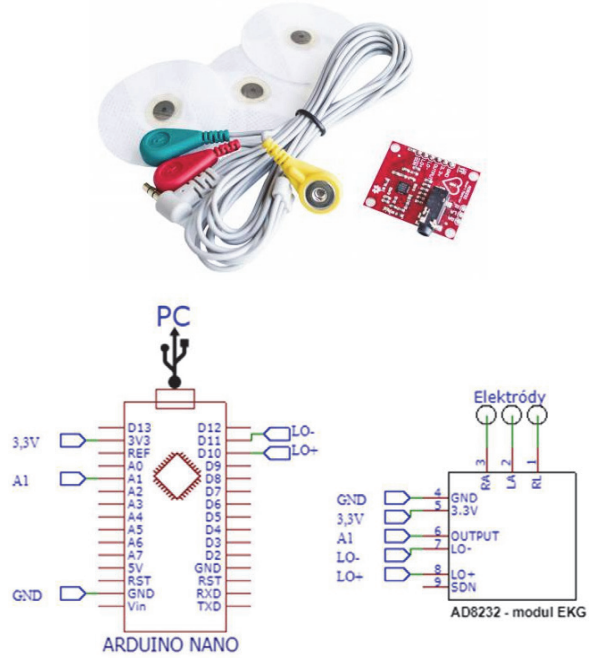

Figure 3: ECG module connection with AD8232 circuit [9].

In this configuration, it is assumed that the patient is at rest during the measurement and tries not to move too much, thus minimizing movement artefacts. The two electrodes are connected to the body according to which lead we want to record. The third electrode is connected to the right limb, which is used as active ground and also serves to suppress interfering signals. In order to be able to measure the ECG curve with minimal distortion, a high-pass filter of the 2 nd order is used in the circuit, which suppresses signals with a frequency lower than $0.5 \mathrm{~Hz}$ and a low-pass filter of the 2nd order, which suppresses signals with a frequency higher than $40 \mathrm{~Hz}$ [9].

Temperature sensor - The thermistor (fig. 4) is a passive semiconductor device whose electrical resistance is temperature dependent. Two types of thermistors are produced, negastors, which heating resistance decreases (NTC) and posistors, which heating resistance increases (PTC). They can have various shapes, most often in the form of plates, drops, beads, rollers. The main advantages of thermistors are high temperature sensitivity, small dimensions, good mechanical resistance and simple implementation of the conversion of electrical resistance to electrical voltage. The disadvantages are a significantly non-linear characteristic, limited temperature range $\left(-40^{\circ} \mathrm{C}\right.$ to $\left.125^{\circ} \mathrm{C}\right)$ and heating due to the passage of the measuring current. We used an
NTC thermistor (fig. 4) from Vishay with a nominal value of $4.7 \mathrm{k} \Omega$ in our device. It has axial outlets to which we connect the supply wires leading to the microcontroller (fig. 4) [10, 11].

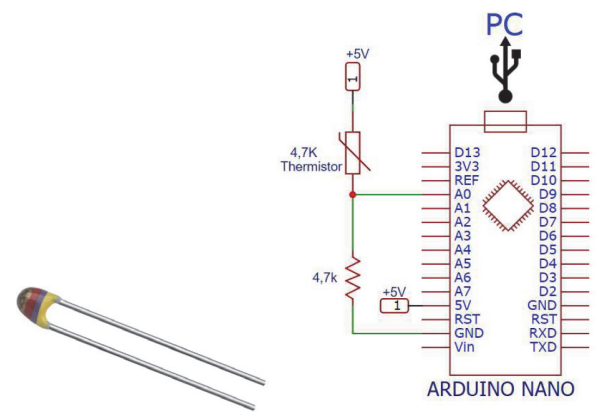

Figure 4: NTC thermistor [15].

The manufacturer states in the datasheet the Steinhart-Hart equation, by which we know from the resistance thermistor to calculate the current temperature [11]:

$$
T_{(R)}=\frac{1}{\left(A_{1}+B_{1} \ln \frac{R}{R_{\text {ref }}}+C_{1} \ln ^{2} \frac{R}{R_{\text {ref }}}+D_{1} \ln ^{3} \frac{R}{R_{\text {ref }}}\right)}
$$

Etalon gauge - pulse oximeter IMDK. This pulse oximeter model is pin-shaped and fits on a finger. Displays the following parameters: blood oxygenation and pulse rate. These are displayed on a two-colour OLED display with dimensions of $25 \times 13 \mathrm{~mm}$. The oximeter even shows the course of the pulse curve. After placing the oximeter on the finger, it displays the resulting values within five seconds, while the measurement is continuous. After removing the finger, it automatically detects its absence and turns off after ten seconds [12].

The built-in LEDs have a wavelength of $660 \mathrm{~nm}$ $( \pm 3 \mathrm{~nm})$ and $904 \mathrm{~nm}( \pm 5 \mathrm{~nm})$. They are connected in antiparallel. The switching frequency is $110 \mathrm{~Hz}$ and each LED is only on for $1 \mathrm{~ms}$. The detection photodiode is located opposite the LEDs, i.e. it is a reflective oximetry. The range of measurement of blood oxygen saturation has $\mathrm{SpO}_{2}=0-100 \%$. Measurement accuracy is $\pm 3 \%$ over the entire measurement range. The heart rate measurement range is $30-240$ BPM with an accuracy of \pm 1 BPM. This device will be further used to verify the functionality of the above sensor systems. 


\section{Design of a device for monitoring human vital signs}

The aim of this work was to design a monitoring device containing functions for monitoring heart rate, body temperature, electrical activity of the heart (electrocardiogram) and blood oxygen saturation. The key module of the device is the development board from Arduino. Arduino is an opensource platform for simple design and development of electronic devices. It is suitable for rapid prototyping and creation of various devices, toys, control and regulation applications or robots. Its use is not conditioned by a deeper knowledge of electronics, which was the main reason why we chose this platform. There are several types of Arduino boards. The electrical connection of all modules with the Arduino is shown in fig. 5 and a proposal of the overall arrangement is shown in fig. 6.
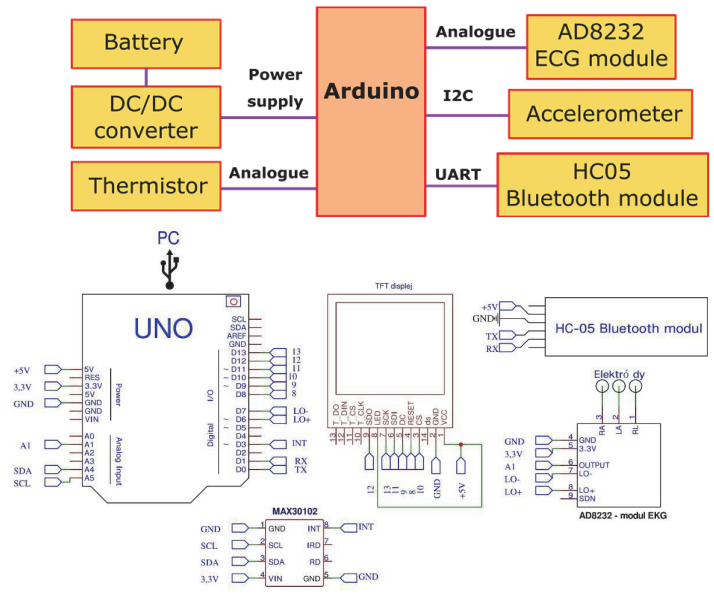

Figure 5: Used modules for device connection.

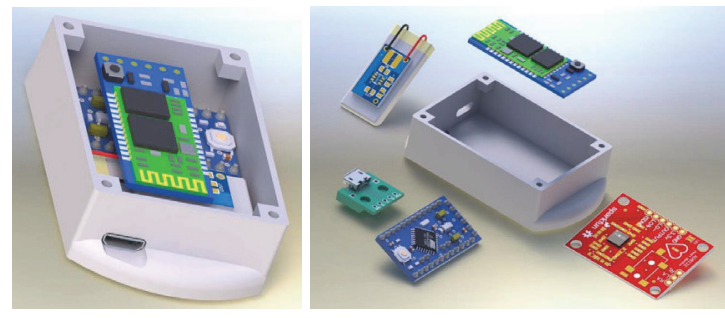

Figure 6: Design of monitoring device layout.

\section{Experimental verification of monitoring equipment functions}

The aim was to compare the results of oximetry between the MAX30102 sensor and a certified oximeter intended for domestic use (IMDK C101H1). The measurement itself (fig. 7) lasted 30 seconds and was performed by simultaneously recording the oxygenation values from both oximeters and then comparing them. The main goal was to compare the accuracy of the measurement and possible deviations.

The measurement procedure was as follows: The subject sat down on a chair and placed both forearms comfortably on the table. The subject placed a certified IMDK C101H1 oximeter on the index finger of the left hand and placed the subject on the MAXIM30102 sensor on the index finger of the right hand. After stabilization of the measured values, which lasts approximately 10-15 seconds, oxygenation values were recorded. The interval between individual measurements was set at a minimum of fifteen minutes.
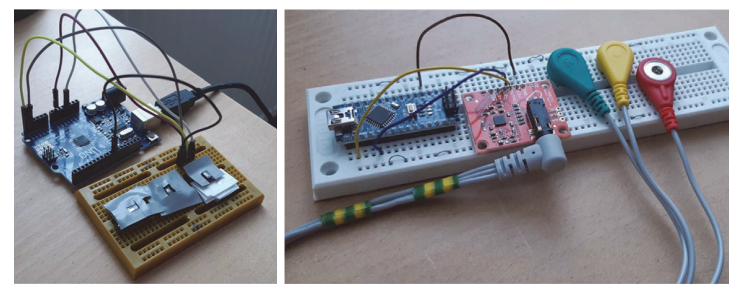

Figure 7: Arduino test connection with MAX30102 sensors and Arduino test connection with AD8232 ECG module.

Further experiments were performed with an electrocardiography module with an AD8232 module connected to an Arduino microcontroller (fig. 7). The aim of the measurement was to obtain the cleanest possible record with a minimum number of artefacts. The measurement on the created electrocardiograph took ten seconds for one lead. This time is long enough for the record to be thoroughly analysed, if necessary.

The measurement was performed as follows: The subject was in a supine position or sitting on a stool. Electrodes were adhered to the subject according to the prescribed rules (on the left and right parts of the chest under the collarbone; approximately ten centimetres from the navel to the left and on the right side so that their joint was as parallel as possible to the thoracic electrode joint). After turning on the serial monitor and initiating communication, an electrocardiographic curve was displayed on the monitor. The functionality of the device was verified for each lead. Limb leads I, II and III were recorded in chronological order. It took ten seconds to measure each lead. 


\section{Results of experiments}

The measurement results of the MAX30102 pulse oximeter module were obtained only after modifying the program library for this module. Based on the measured data, Arduino then evaluated the value of blood oxygenation. Fig. 8 shows the results of individual measurements. Data measured over thirty seconds were approximated by arithmetic mean and rounded to one decimal place. From this graph it can be seen that the oxygenation values differed only minimally. The largest deviation recorded was $0.9 \%$, which is still the norm because the pulse oximeter manufacturer states an accuracy of $\pm 3 \%$.
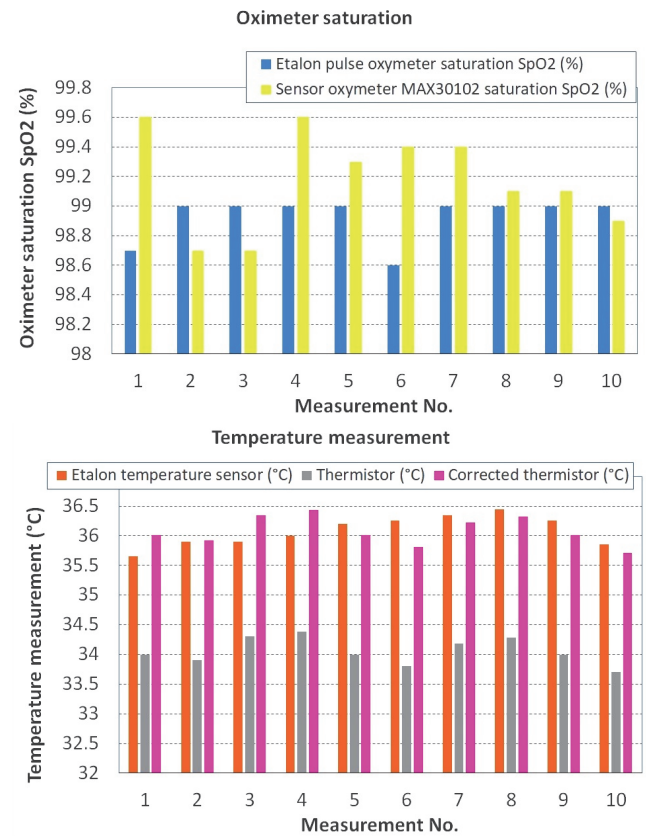

Figure 8: Results of measuring oxygen saturation $\mathrm{SpO}_{2}$ and results of human body temperature measurement.

When measuring the temperature with a thermistor, the values differed significantly from the reference standard. All measured values are shown in the graph of fig. 8. We can see that the temperature measured by the thermistor deviated from the reference values. This temperature was always lower by a certain amount. The deviations ranged from 0.84 to $2.45^{\circ} \mathrm{C}$. Since these values have the same tendency, it is possible to correct these deviations by means of a constant coefficient and it is possible in part to reduce these measurement deviations in this way. The correction coefficient was determined with a value of 1.06 and the values measured by the thermistor can be multiplied by this coefficient. After this correction, the maximum deviation of the measured temperature values fell below $0.45^{\circ} \mathrm{C}$, which represents $1.2 \%$ of the nominal value.

Pulse measurement was performed by two methods (fig. 9) using modules MAX30102 and AD8232. A pulse oximeter was used as a reference instrument. The measurements lasted 30 seconds.
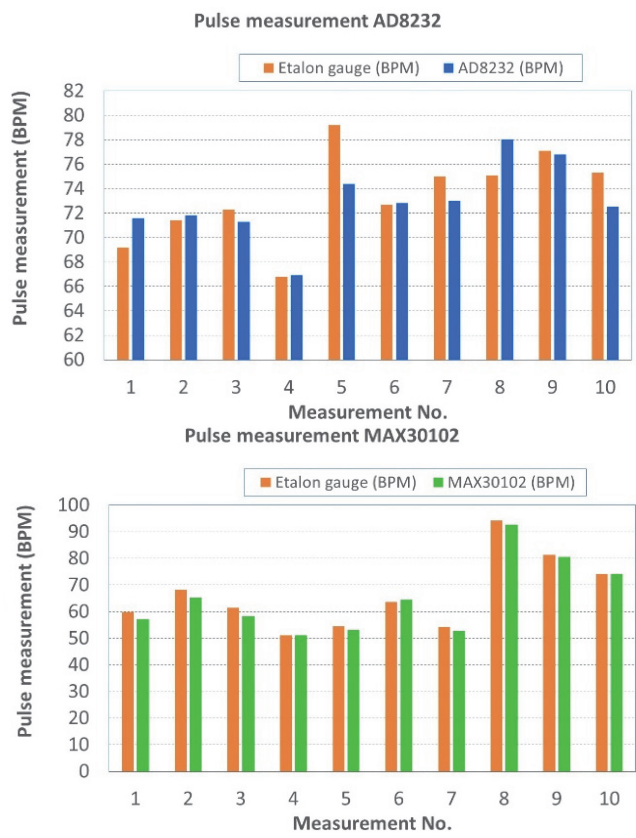

Figure 9: Pulse measurement using sensors.

For the AD8232 ECG module, the method for determining the time between two consecutive $R$ oscillations was used to calculate the pulse. For this reason, the individual measurements (fig. 9) showed a wide range of pulse values, but their average value was approximately the same compared to the pulse oximeter. The graphic records show the differences between the values determined by the sensors and the values determined by the selected reference meter. The maximum deviation was determined for the AD8232 sensor with a value of 4.8 BPM which is $6 \%$ of the nominal value and for the MAX30102 sensor the maximum deviation was determined with a value of $3.2 \mathrm{BPM}$ which is $5.3 \%$ of the nominal value determined by the selected reference meter. Thus, both sensors showed qualitatively approximately the same measurement results (fig. 9).

ECG measurement - The use of a dry electrode 
chest strap with an ECG module in a two-electrode circuit was not the best solution (fig. 10).

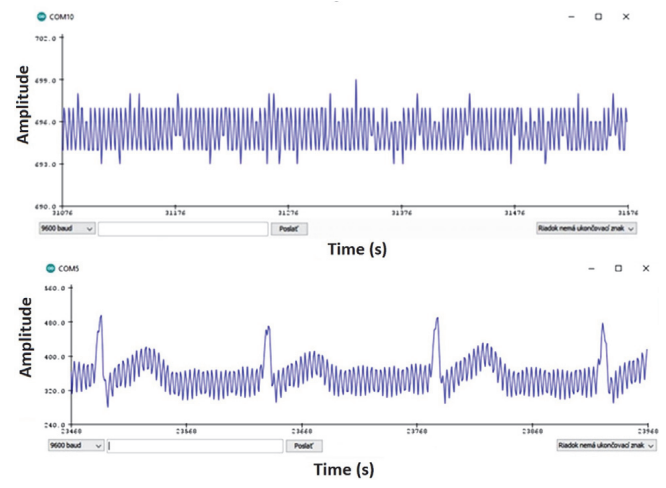

Figure 10: Example of measurement with a desktop computer and Example of measurement with a laptop without network connection.

The AD8232 integrated circuit is wired in a configuration for use with three measuring electrodes, not two. Therefore, obtaining the measured data was problematic. When connecting the Arduino to a desktop computer, the measured values were unusable, there was probably network interference. An example of such a measurement is shown in fig. 10. However, if we connected the Arduino to a laptop that was powered by a battery, the measured values looked a little more readable, although it was still not optimal. An example of this measurement is shown in fig. 10. What the measurement looks like when the third electrode is connected to the right hand is shown in fig. 11.

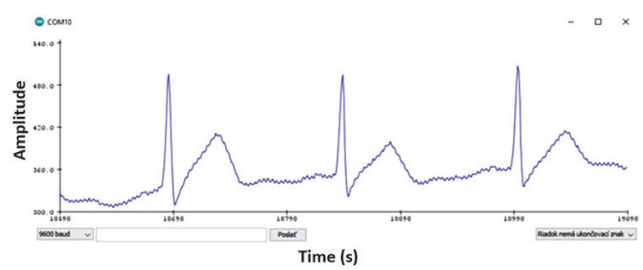

Figure 11: Example of measurement with a desktop computer and Example of measurement with a laptop without network connection.

\section{Conclusions}

The results of measurements of individual quantities are satisfactory and can be used for approximate domestic measurements intended for preliminary diagnosis of the patient. By integrating all the above-mentioned measuring systems, it is thus possible to obtain a device for monitoring human vital signs. The measurement of human respiratory rate and blood pressure is expected to be extended in the future. Another planned part of the development is the monitoring of human physical activities and the overall regime of his activities. The stage of the solution also includes the recording of measured data and their processing for the purpose of determining further drug care for the patient. The field of application is mainly in domestic non-professional conditions as a prevention against collapse conditions of the patient. The device is assembled with low-cost elements and so the resulting price is favourable. The overall measurement results were confronted only with the usual cheap available meters and still need to be verified with professional meters $[13,14,15]$.

\section{Acknowledgments}

The authors would like to thank the Slovak Grant Agency KEGA 030TUKE-4/2020 "Transfer of knowledge from the field of industrial automation and robotics to the education process in the teaching program mechatronics" and project KEGA 016TUKE-4/2021 "New educational technologies of metrologists for monitoring of production processes and post-processing of products". Research of new measuring methods of machine condition. This paper was published in cooperation with company KYBERNETES Ltd. within the project "Research and development of the ECOGI product at KYBERNETES", ITMS Code of Project: 3130120955 .

\section{References and Notes}

1. Cunha, J. P. S., Cunha, B., Pereira, A. S., Xavier, W., Ferreira, N. and Meireles, L.: "Vital-Jacket ${ }^{\oplus}$ (2010). A wearable wireless vital signs monitor for patients' mobility in cardiology and sports. 2010 4th International Conference on Pervasive Computing Technologies for Healthcare, 2010, Date of Conference: 22-25 March 2010. Conference Location: Munich, Germany. Publisher: IEEE. pp. 1-2, doi: 10.4108/ICST. PERVASIVEHEALTH2010.8991.

2. He, D. D., Winokur, E. S. And Sodini, C. G. (2015). An Ear-Worn Vital Signs Monitor. In IEEE Transactions on Biomedical Engineering, vol. 62, no. 11, Publisher: IEEE, pp. 2547-2552, Nov. 2015, doi: 10.1109/TBME.2015.2459061.

3. Weller, R.S., Foard, K.L. \& Harwood, T.N. (2018). Evaluation of a wireless, portable, wearable multi-parameter vital signs monitor in hospitalized neurological and neurosurgical patients. J Clin Monit Comput 32, pp. 945-951 (2018). https://doi.org/10.1007/s10877-017-0085-0.

4. Winokur, E. S., He, D. D. And Sodini, C. G. (2012). A wearable vital signs monitor at the ear for continuous heart rate and 
Pulse Transit Time measurements. 2012 Annual International Conference of the IEEE Engineering in Medicine and Biology Society, Date of Conference: 28 Aug.-1 Sept. 2012, Conference Location: San Diego, CA, USA, 2012, Publisher: IEEE, pp. 2724-2727, doi: 10.1109/EMBC.2012.6346527.

5. SHU, M., TANG, M., YANG, M. AND WEI, N. (2017). The Vital Signs Real-Time Monitoring System Based on Internet of Things. 2017 4th International Conference on Information Science and Control Engineering (ICISCE), Date of Conference: 21-23 July 2017, Conference Location: Changsha, China, Publisher: IEEE, 2017, pp. 747-751, doi: 10.1109/ICISCE.2017.160.

6. Pulse Sensor [online]. [cit. 2021-5-14]. Available online: https://pulsesensor.com/.

7. Maxim Integrated MAX30102 Pulse Oximeter \& HeartRate Sensor. Mouser [online]. [cit. 2021-5-14]. Available online: https://eu.mouser.com/new/maxim-integrated/ maximmax30102efd-sensor/

8. HAMPTON, J. R., HAMPTON, J. (2019). The ECG Made Easy E-Book. 9th edition. Elsevier Health Sciences, 2019. pages 240. ISBN 0702075043, 9780702075049.

9. Analog Devices. "Single-Lead, Heart Rate Monitor Front End," AD8232 datasheet, Aug. 2012.

10. GENEVA, I. I., CUZZO, B., TASADUQ FAZILI, T., JAVAID, W. (2019). Normal Body Temperature: A Systematic Review. In. Open Forum Infectious Diseases. Volume 6, Issue 4, April 2019, ofz032, pp. 1-7. https://doi.org/10.1093/ofid/ofz032.

11. VISHAY, "NTC Thermistors, Radial Leaded, Standard Precision," 29049 Datasheet, Jan. 2021.

12. IMDK C101H1 pulse oximeter. (2021). User guide. IMDK Medical technology CO., Ltd.

13. Belvončíková, D., Bednarčíková, L., Michalíková, M., Štefanovič, B., Trebuňová, M., Mezencevová, V., \& Živčák, J. (2020). Development of Mechanism for Finger Prosthesis. Acta Mechanica Slov., 24(4), 6-11. doi: 10.21496/ ams.2020.028.

14. Živčák, J., Rajtúková, V., Krajňáková, V., Hudák, R., \& Kátlovská, D. (2020). Individual Lower Limb Orthosis. Acta Mechanica Slov, 24(3), 6-9. doi: 10.21496/ams.2020.024.

15. Mitrík, L., Klímová, J., Trebuňová, M., Schnitzer, M., Hudák, R., Živčák, J., Molnár, L., \& Modrák, M. (2020). Pre-clinical Testing of the Polymer Implants Enriched of Ceramic Materials with aim on Rate of Osteointegration in a Rabbit Model. Acta Mechanica Slov., 24(1), 14-18. doi: 10.21496/ams.2020.013

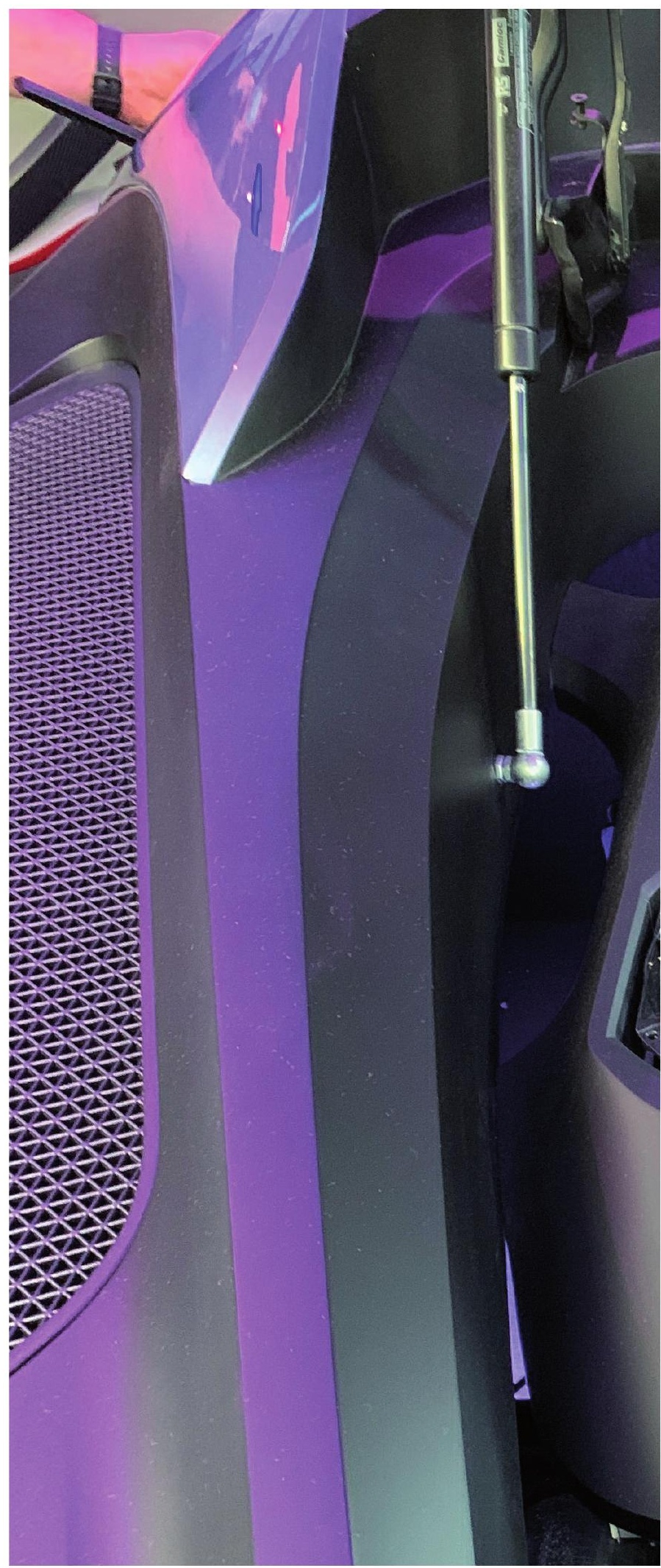

